\title{
Spezifische Investitionen als Legitimationsgrundlage für Stakeholderansprüche
}

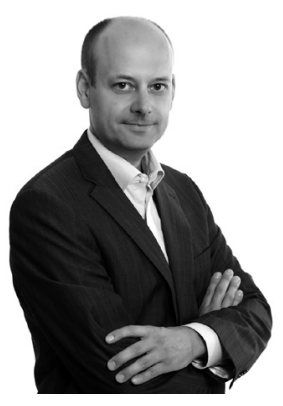

\author{
Alexander Brink
}

Theorie der Unternehmung, spezifische Investitionen, Teamproduktionstheorie, Theorie unvollständiger Verträge, Stakeholdermanagement

Theory of the firm, specific investments, team production theory, theory of incomplete contracts, stakeholdermanagement

Im Zentrum des vorliegenden Beitrags steht die Frage, welche unternehmerische Anspruchsgruppe ein residuales Risiko trägt und damit einen begründeten Anspruch auf das unternehmerische Residuum

hat. Im Detail möchte ich auf Basis der Theorie spezifischer Investitionen, der Teamproduktionstheorie und der Theorie unvollständiger Verträge eine Erweiterung der Theorie der Unternehmung vorschlagen. Das Unternehmen wird zunächst - am Beispiel des Mitarbeiters - vertragstheoretisch rekonstruiert, sodann genauer als Vertragsbündel ungesicherter spezifischer Investitionen. Ungesicherte spezifische Investitionen stellen eine Legitimationsgrundlage für Residualansprüche dar. Ein Anstieg der spezifischen Investitionen bewirkt eine Zunahme der impliziten Verträge, die sich ex ante vertraglich kaum absichern lassen. Mit zunehmender Implizität der Verträge steigen damit auch die Ansprüche des Mitarbeiters. Die klassischen beiden Absicherungsmechanismen, Eigentum und Kontrolle, werden durch eine dritte Variante ergänzt: das sich aus den treuhänderischen Pflichten ableitende Versprechen des Boards. Aus den zentralen Problemen mit Blick auf Ansprüche von unspezifisch investierten Stakeholdern und non-Stakeholdern ergibt sich weiterer Forschungsbedarf.

This paper focuses on the following question: Which stakeholder group bears residual risk and can therefore raise a reasonable claim on enterprise residuum? Specifically, this paper seeks to build on team production theory and specific investments in order to propose an extension to the theory of the firm. Beginning with the example of an employee, the enterprise will be reconstructed from the perspective of contract theory, and presented then more precisely as a contract bundle of unsecured specific investments. Unsecured specific investments represent a basis for the legitimation of residual claims. A rise in specific investments effects an increase in implicit contracts which can hardly be secured contractually ex ante. As the implicitness of these contracts rises there is also a correlated rise in employee claims. The two classical safeguards, ownership and control, are then supplemented by a third variant: the promise of boards which arises from their fiduciary duties. This central problem, with its focus on the claims of non-specifically invested stakeholders as well as non-stakebolders, exposes a need for further research. 


\section{Einleitung}

In der internationalen Literatur zu Corporate Governance wird die Legitimation von Stakeholderansprüchen auf das unternehmerische Residuum vielfältig begründet (Jensen/Meckling 1976; Bowie/Freeman 1992; Hill/Jones 1992; Quinn/Jones 1995; Suchman 1995; Phillips 2003). Im Verhältnis zueinander werden die beiden wichtigsten Theoriestränge, die Agenturtheorie und die Stakeholdertheorie, als „polar opposites“ (Shankman 1999, 319) charakterisiert. ${ }^{1}$ In der wissenschaftlichen Auseinandersetzung über eine Auflösung dieses vermeintlichen Gegensatzes sind gegenwärtig zwei Positionen in der Diskussion: Aus der Kritik an einer einseitig ökonomischen Interpretation von Agenturtheorien heraus argumentieren einige Managementtheoretiker wie etwa Ghoshal (2005) für eine stärkere Stakeholderorientierung (vgl. auch Ghoshal/Moran 1996). Andere versuchen den ökonomischen Kern bzw. die ökonomische Relevanz von Stakeholdertheorien zu verdeutlichen (Freeman 1984; Jones 1995; Jensen 2001). Keinem der beiden Lager gelingt jedoch eine systematische Zusammenführung von Agentur- und Stakeholdertheorie: Im ersten Fall erfolgt eine normative Korrektur der ökonomischen Perspektive, im zweiten Fall eine Funktionalisierung des Stakeholderansatzes. Es bleibt also ein Forschungsdesiderat zu zeigen, dass eine umfassende Stakeholderperspektive aus der Agenturtheorie governancetheoretisch dargestellt werden kann. ${ }^{2}$ Versteht man das Unternehmen zum Beispiel als Netzwerk von Verträgen, also als multiple Prinzipal-Agent-Beziehung, so ist die Berücksichtigung von legitimen Stakeholderansprüchen governancetheoretisch sogar zwingend notwendig: „In this way, stakeholder theory is argued to be the logical conclusion [sic!] of agency theory. "Shankman 1999, 319)

Dem vorliegenden Beitrag liegt also eine governancetheoretische Rekonstruktion von Stakeholdermanagement zugrunde. Im Zentrum der systematischen Vereinbarkeit von Agenturund Stakeholdertheorien geht es um die Frage, welche Anspruchsgruppe ein residuales Risiko trägt und damit einen begründeten Anspruch auf das Residuum hat. Im Detail möchte ich auf Basis der Teamproduktionstheorie, der Theorie spezifischer Investitionen und der Theorie unvollständiger Verträge eine Erweiterung der Theorie der Unternehmung diskutieren. Dabei wird ein besonderer Fokus auf die Stakeholdergruppe der Mitarbeiter gelegt und deren Bedeutung im Vergleich zu den Aktionären gestärkt.

Zunächst wird die Theorie der Unternehmung aus einem traditionellen Verständnis heraus vertragstheoretisch dargestellt (Kapitel 2). Sodann wird das Unternehmen genauer als Vertragsbündel ungesicherter spezifischer Investitionen verstanden. Ich werde erläutern, warum ungesicherte spezifische Investitionen eine Legitimationsgrundlage für Residualansprüche darstellen (Kapitel 3). In einem weiteren Schritt werde ich drei Absicherungsmechanismen - Eigentum, Kontrolle und Versprechen - kritisch erläutern (Kapitel 4) und schliesslich das sich aus den treuhänderischen Pflichten ableitende Versprechen des Boards (Kapitel 5). Im Ergebnis steht eine governancetheoretische Position, nach der Anspruchsgruppen immer dann legitime Ansprüche auf das Residuum haben, wenn sie ihre spezifischen Investitionen nicht absichern können.

Durch die hier vorgestellte Absicherungstrias aus Eigentum, Kontrolle und Versprechen kann der von einigen Unternehmensethikern häufig eingeforderten Übernahme von korpo-

1 Eine ähnliche Einschätzung findet sich bei Quinn/Jones (1995, 22). Vgl. zum Stakeholderansatz vertiefend Freeman 1984; Jones 1995; Freeman/Reed 1983 und Donaldson/Preston 1995; in Bezug auf die Agenturtheorien vgl. insbesondere McCall 2001; Friedman 1970 oder Heath/Norman 2004.

2 Vgl. auch diesbezügliche Positionen bei Hill/Jones 1992 [stakeholder-agency theory]; Clarkson 1995 [risk based model of stakeholder theory]; Quinn/Jones 1995 [agent-morality approach] und Shankman 1999. 
rativer Verantwortung bis zu einem gewissen Grade institutionenökonomisch und vertragstheoretisch Rechnung getragen werden (Thomsen 2004). Zwar hat ein solcher Ansatz den Charme der ökonomischen Anschlussfähigkeit, es bleiben aber zentrale Probleme bestehen mit Blick auf Ansprüche von unspezifisch investierten Stakeholdern und non-Stakeholdern. Hieraus ergibt sich weiterer Forschungsbedarf, an dem sich auch andere nichtökonomische - u.a. normative - Disziplinen beteiligen müssen (Kapitel 6).

\section{Das Unternehmen als Vertragsbündel}

Jensen/Meckling (1976) beschreiben in Anlehnung an Alchian/Demsetz (1972) das Unternehmen als Netzwerk bilateraler Verträge (nexus of bilateral contracts). Im Fokus steht der Vertrag,

„[...] under which one or more persons (the principal[s]) engage another person (the agent) to perform some service on their behalf which involves delegating some decision making authority to the agent" (Jensen/Meckling 1976, 308).

Dieser vertragstheoretische Grundgedanke wurde in zwei Ansätzen auf mehrere Anspruchsgruppen ausgedehnt: Zum einen wird das Unternehmen nach Freeman/Evan (1990) als „set of multilateral contracts over time“ (Jensen/Meckling 1976, 352) verstanden. Im Unterschied zur traditionellen Stakeholdertheorie, bei der die Legitimation von Ansprüchen primär aus den Konsequenzen unternehmerischer Transaktionen abgeleitet wird (Freeman 1984), ergeben sich nunmehr legitime Ansprüche aus einer einvernehmlichen vertraglichen Lösung. Vertragliche Arrangements zur gegenseitigen Wertsteigerung beinhalten nicht nur explizite, sondern auch implizite Verträge. Ein impliziter Vertrag schliesst sämtliche Bestandteile in oder neben einem expliziten Vertrag ein, die nicht präzise ausgehandelt und kodifiziert wurden, deren Änderung oder Herausnahme aber mindestens eine der Parteien den Vertrag nicht hätte schliessen lassen.

Zum anderen haben Hill/Jones (1992) den vertragstheoretischen Ansatz zu einer StakeholderAgency-Theorie ausgearbeitet, der zufolge das Management die Funktion als Agent verschiedener Anspruchsgruppen übernimmt (vgl. auch Quinn/Jones 1995): „(E)ach stakeholder is a part of the nexus of implicit and explicit contracts that constitutes the firm. " (Hill/Jones 1992, 134) Je mehr Stakeholder sich an diesem Netzwerk beteiligen, desto eher werden die Interessen der Anspruchsgruppen ausbalanciert.

Vor diesem Hintergrund möchte ich Oliver Williamson hinzuziehen, der für den im Folgenden nachzuzeichnenden vertragstheoretischen Ansatz einen geeigneten Ausgangspunkt liefert (Williamson 1998, 36; Williamson 2002, 173). Williamson macht seine vertragstheoretische Position mit einem Zitat von James M. Buchanan deutlich (vgl. dazu auch Buchanan 1964 und 1975):

„(E)conomics comes closer to being a ,science of contract ${ }^{6}$ than a ,science of choice $[$ [...] [on which account] the maximizer must be replaced by the arbitrator, the outsider who tries to work out compromises among conflicting claims." (Buchanan 1975, 229)

Die Designs dieser Verträge variieren hinsichtlich der Kosten, wobei sich die relative ökonomische Vorteilhaftigkeit der Verträge aus den Eigenschaften einzelner Transaktionen ergibt. ${ }^{3}$

3 In der Williamson'schen Terminologie sind Häufigkeit (frequency), Unsicherheit (uncertainty) und Spezifität (asset specificity) die Variablen zur Bestimmung der Höhe von Transaktionskosten. Die Spezifität gilt für Williamson als entscheidende Einflussgrösse (Williamson 1984b, 202ff.). 
Damit verdrängt die Transaktion als „basic unit of analysis“ (Williamson 1984a, 1229) die individuelle Entscheidung, wie sie etwa mit dem homo oeconomicus dem methodologischen Individualismus in der Neoklassik zugrunde liegt. Zugleich bedient sich Williamson eines Argumentes von Chester I. Barnard (1938), Governance nicht als Macht-, sondern als Konsensphänomen zu betrachten (Williamson 1990/1996, 31).

„(T)he consensual view of authority - more generally, the consensual view of contract - has been enormously influential for reconceptualizing the study of economic organization. " (Williamson 1990/1996, 32)

Eine Theorie der Unternehmung wird also nach Williamson als Vertragsproblem verstanden, bei dem es um Konsenslösungen durch Verhandlungen geht (Williamson 1985, 20). ${ }^{4}$

\section{Spezifische Investitionen des Mitarbeiters als Legitimationsgrundlage für Residualansprüche}

Auf Basis der zuvor gewonnenen Erkenntnisse werde ich das Unternehmen nunmehr präziser als Vertragsnetzwerk spezifischer Investitionen nachzeichnen (Rajan/Zingales 1998; Zingales 1998; Blair/Stout 1999; Tirole 1999; Roberts/van den Steen 2000; Zingales 2000; Tirole 2001). Spezifität liegt immer dann vor, wenn eine Transaktion durch Ressourcen unterstützt wird, deren Wert durch alternative Widmung abnimmt. ${ }^{5}$ Faktorspezifität bezieht sich auf den Grad der Wiederverwendbarkeit eines bestimmten Vermögensobjektes in alternativen Investitionen ohne Verlust an Produktionswert.

"(A)sset specificity refers to durable investments that are undertaken in support of particular transactions, the opportunity cost of which investments is much lower in best alternative uses or by alternative users should the original transaction be prematurely terminated." (Williamson $1984 b, 202$ )

Die Differenz der Wertschöpfung zwischen einer spezifischen und der nächstbesten Investition eines Faktors wird auch als transaktionsspezifische Quasirente bezeichnet. Die Höhe dieser Quasirente bestimmt folglich das Mass der Spezifität eines Faktors. Der Erfolg des Netzwerkes ist die Summe der den spezifischen Investoren zurückfliessenden Renten. Spezifische Investitionen und Quasirenten einzelner Anspruchsgruppen sind riskant, weil sie ausgebeutet werden können und damit „costly contractual breakdowns“ (Williamson 2002, 174) drohen. Diese Ausbeutung wird auch als hold-up bezeichnet und ist eine Sonderform des auf dem Opportunismus basierenden moral hazards (Ross 1973; Furubotn/Richter 2005). Ziel ist es daher, potentielle Interessenkonflikte zwischen den Vertragspartnern zu verhindern und frühzeitig Absicherungen (contractual and organizational safeguards) $\mathrm{zu}$ suchen (Williamson 1984b, 202). Da sich die Bedeutung firmenspezifischer Investitionen im Zeitablauf ändern kann, sind Absicherungen immer eine Verhandlungssache. ${ }^{6}$

4 Obwohl nicht alle Beziehungen tatsächlich vertraglich geregelt werden, kann man sich auf den Standpunkt stellen, „as if they were sets of contracts“ (Clark 1985, 62). Dies erklärt, warum auch andere Anspruchsgruppen explizit als Vertragspartner einzubinden sind (zur Vertiefung Jensen/Meckling 1976, 310; Hill/Jones 1992).

5 Williamson (1985, 95ff.) unterscheidet vier verschiedene Formen von Spezifität: (1) site specificity bezieht sich auf die wechselseitige Abhängigkeit von Käufer und Verkäufer, (2) physical asset specificity greift z.B. die Investition in Maschinen auf, (3) buman capital specificity nimmt spezifisches Know-how in den Blick und (4) die dedicated assets umfassen allgemeine Investitionen. Im vorliegenden Beitrag steht das Humankapital im Zentrum.

6 So können sich z.B. bei Mergers \& Acquisitions nachträgliche Verhandlungsoptionen (renegotiations) ergeben (Tirole 1999). 
„These firm-specific investments create room for bargaining [sic!] and have to be governed after [sic!] the contracts have been made. For this reason, corporate governance can be defined as a set of constraints shaping the ex post bargaining over the joint output of firm-specific investments." (Osterloh/Frey 2005b, 10)

Ein Anstieg der spezifischen Investitionen bewirkt nicht selten auch eine Zunahme der impliziten Verträge. Dadurch wird eine fundamentale Transformation der Verhandlungssituation eingeleitet (Williamson 1985). Risiken werden nunmehr durch bestimmte Governancemechanismen abgesichert. Es geht also um die Verhandlung über Absicherungen gegen potentielle Ausbeutung bei spezifischer Investition einzelner Stakeholder. Ein Stakeholder wird in diesem Kontext in Anlehnung an die Teamtheorie mit Wieland definiert als

„[...] ein Ressourcenbesitzer in einem durch explizite und implizite Verträge konstituierten, kooperativen Team, dessen Zweck die Erwirtschaftung einer Kooperationsrente durch die Realisierung einer distinkten Transaktion mittels einer angemessenen und dauerhaften Governanceform ist" (Wieland 2008, 25).

Da jedoch nicht alle spezifischen Investitionen abgesichert werden können, bleiben vielfältige und konfliktäre Residualansprüche bestehen (Sadowski et al. 2003, 149).

Die Führung, Steuerung und Kontrolle eines solchen Vertragsnetzwerks spezifischer Investitionen kann man grob unter den Begriff der Netzwerkgovernance fassen (Calton/Lad 1995; Jones et al. 1997; Wieland 2007). Netzwerkgovernance ist den Williamson'schen hybriden Governanceformen zuzuordnen und damit eine moderne Alternative zu Markt und Hierarchie, die Transaktionskostentheorie und soziale Netzwerktheorie miteinander verbindet (Coase 1937).

„Network governance involves a select, persistent, and structured set of autonomous firms [...] based on implicit and open-ended contracts to adapt to environmental contingencies and to coordinate and safeguard exchanges." (Jones et al. 1997, 914)

Corporate Governance ist in diesem Sinne „the complex set of constraints that shape the ex post bargaining between finance and management over the quasi-rents generated by a firm “ (Zingales 1998, 498). Die meisten Ökonomen gehen nunmehr davon aus, dass allein Shareholdern ein Anspruch auf das Residuum zusteht, da sie allein das residuale Risiko tragen und ihre Risiken als einzige Anspruchsgruppe nicht absichern können (Williamson 1984a, 1213ff.; Hansmann 1996; Shleifer/Vishny 1997). Alle anderen Anspruchsgruppen werden über explizite Verträge abgesichert. ${ }^{7}$ Williamson etwa schreibt: „The whole of their [the shareholders', A. B.] investment in the firm is potentially placed at hazard. " (Williamson 1984a, 1209; Williamson 1985, 304) Und er erklärt den Zusammenhang noch ausführlicher:

„Stockholders as a group [sic!] bear a unique relation to the firm. They are the only voluntary constituency whose relation with the corporation does not come up for periodic renewal. Labor, suppliers in the intermediate product market, debt-holders, and consumers all have opportunities to renegotiate terms when contracts are renewed. Stockholders, by contrast, invest for the life of the firm and their claims are located at the end of the queue should liquidation occur." (Williamson 1984a, 1210)

7 Clarkson (1995) verweist in einem Beitrag darauf, dass auch andere Stakeholder ähnliche Risiken wie Shareholder (Investitionsrisiko, Dividendenausfall, Konkurs) eingehen, so etwa Gläubiger (Schuldenausfall), aber auch Anbieter und Kunden. Allerdings sind diese Risiken zum Grossteil bereits geschützt, lediglich bei den Mitarbeitern bleiben ungesicherte Risiken. 
Boatright kommt zu ähnlichen Ergebnissen:

„For shareholders this means that they will receive all residual revenues and that the firm will be operated to maximize the residual. For employees this means that wages will be paid, that they will receive some portion of the quasi-rents created by their acquisition of firm-specific knowledge and skills, and that they will share in the prosperity of the firm. " (Boatright 2004, $13)^{8}$

Während Williamson und Boatright (und im Übrigen auch Zingales 1998 und 2000) die Quasirenten der Aktionäre und des Managements in den Blick nehmen, möchte ich im vorliegenden Beitrag auch andere spezifisch investierte Anspruchsgruppen (insbesondere die Mitarbeiter) in die ex-post-Verhandlungen einbeziehen. ${ }^{9}$ Obwohl eine quantitative Bestimmung des spezifischen Humankapitals äusserst diffizil ist, ${ }^{10}$ finden sich mindestens drei positive Effekte, die die Relevanz spezifischen Humankapitals unterstreichen: So steigt die Vergütung der Mitarbeiter in der Regel stärker mit der Dauer der Firmenzugehörigkeit, als man dies durch einen Zuwachs an unspezifischem Humankapital erklären kann. Ferner sinkt die Fluktuationsrate bei Unternehmen mit hohem spezifischen Humankapital: Es gibt sowohl weniger Entlassungen als auch weniger freiwillige Kündigungen. Und schliesslich steigt der Einkommensverlust von Mitarbeitern nach betriebsbedingten Kündigungen, die lange im jeweiligen Unternehmen gearbeitet und ein hohes spezifisches Humankapital gebildet haben (Blair 1995, 263ff.).

Die Wirkung der spezifischen Investitionen auf die Wertschöpfung ist im Detail schwer zu operationalisieren. Dennoch stellt sich die Frage, wann eine Absicherung für das Unternehmen profitabel ist. Es liegt die Vermutung nahe, dass bei einer Nicht-Absicherung die

8 Dieser Teil scheint insofern kritisch, als die Teilhabe am unternehmerischen Erfolg, die Boatright hier einfordert, letztlich - sofern sie ex post gewährt wird (und das ist in der Regel der Fall, da Erfolg nur ex post zu messen ist) - das Residuum mindert. Folglich hat auch der Mitarbeiter letztlich einen Anspruch auf das Residuum, obwohl Boatright sich deutlich von einem residualen Anspruch der Mitarbeiter auf das Residuum distanziert: „(E)mployees have no claim on residual revenues.“ (Boatright 2004, 13) Im Gegensatz zu Boatright wird in dem hier vorliegenden Beitrag nicht nur von einem allgemeinen Risiko, sondern von einem residualen Risiko ausgegangen, also einem Risiko, das ex ante nicht vertraglich über Vergütung kompensiert werden kann. Boatright versucht diese Unklarheit aufzulösen, indem er den Mitarbeitern variable revenues zugesteht: „Employees are often motivated with an implicit understanding that wages will rise with revenues. Sometimes this agreement is explicit in the form of productivity bonuses. Thus, employees have some claim on a firm's variable revenues that is similar to the shareholders' claim on residual revenues. " (Boatright 2004, 13)

9 Am Beispiel des contracting schema von Williamson kann man vier Fälle unterschiedlicher Vertragsdesigns beschreiben (Williamson 2002, $182 \mathrm{ff}$., sowie Williamson 2005, 9ff.). Bei einer unspezifischen Investition des Mitarbeiters wählen die Vertragspartner als Governancemechanismus die Marktlösung, da sie an einer vertraglichen Absicherung aufgrund der fehlenden hold-up-Gefahr kein Interesse haben (unassisted market). Sichert ein Mitarbeiter eine spezifische Investition nicht ab, stellt die Abhängigkeit vom Vertragspartner ein Risiko für den Mitarbeiter dar, das über eine höhere Prämie kompensiert wird (unrelieved hazard). Handelt es sich um eine langfristige Beziehung zwischen Unternehmen und Mitarbeiter, so sind Nachverhandlungen wahrscheinlich, so dass spezielle Governancestrukturen geschaffen werden wie z.B. Abfindungen bei Entlassungen oder interne Klagesysteme. Unterliegen beide Seiten, also Arbeitgeber und Mitarbeiter, aufgrund co-spezifischer Investitionen der Gefahr eines wechselseitigen hold-ups und sind die jeweiligen spezifischen Investitionen sehr hoch, werden die vertraglichen Absicherungen verstärkt (credible contracting) (Che/Hausch 1999). Bei anderen Stakeholdern wie z.B. Kunden oder Lieferanten würde als „form of last resort“ (Williamson 2002, 183; Williamson 2005, 12) auch eine komplett vertikale Integration in Erwägung gezogen (bierarchy), für Mitarbeiter ist diese Variante allerdings nicht möglich (Klein et al. 1978). Im vorliegenden Beitrag geht es also insbesondere um die dritte Variante, das credible contracting.

10 Gathmann/Schönberg etwa forschen empirisch zur Bedeutung von spezifischen Humankapitalinvestitionen. Dabei untersuchen die beiden Autorinnen die Mobilität des spezifischen Humankapitals und dessen Einfluss auf die individuelle Entlohnung (Gathmann/Schönberg 2010, insbesondere 16ff.). Vgl. auch grundlegend Becker 1964. 
spezifische Investition in Humankapital völlig unterbleibt, da der Mitarbeiter die hold-upGefahr antizipiert und das Ausbeutungsrisiko scheut. Damit läge ein negativer Effekt auf die Kooperationsrente und die Effektivität und Effizienz des Teams vor. Aus einer unternehmenswertorientierten Perspektive ist eine Absicherung spezifischer Investitionen für das Unternehmen immer dann ökonomisch sinnvoll, wenn die Kosten für die Absicherung spezifischer Investitionen - also die Kosten für die Beteiligung am Eigentum bzw. an der Kontrolle oder aber die Kosten für die Einhaltung von Versprechen - nicht über dem Wert der transaktionsspezifischen Quasirente liegen.

Ein kritischer Risikovergleich verschiedener Kriterien wie Diversifizierung, Investitionstyp, Investitionsmenge, Risikohöhe und Sicherheit macht deutlich, dass Aktionäre gegenüber dem Mitarbeiter zum Teil deutlich geringere Risikograde aufweisen, als man prima facie glauben mag. ${ }^{11}$ Dass Aktionäre generell weniger risikoavers sind als Mitarbeiter, stützt diese Beobachtung. Auf die besondere Risikoanfälligkeit von Mitarbeitern wird von einigen Autoren verwiesen (Fontrodona/Sison 2006; Soppe 2008; aber auch Blair 1995 und 2003). Soppe (2008) kommt zu dem Ergebnis, dass das (residuale) Risiko des Mitarbeiters im Vergleich zu anderen Stakeholdern sogar am höchsten ist.

„The downside risk of the employee is considered the most severe of all stakeholders. Because of long term education and long term fixed contracts, employees have a vulnerable bargaining position in the sticky labour market. Most important is the fact that employees cannot easily diversify their labour contracts. This makes the individual employee the most vulnerable party in this stockholder's approach of the market economy. “ (Soppe 2008, 218)

\section{Zu einer ähnlichen Feststellung kommt auch Blair:}

„If the claims of all other participants are fully protected by contract, according to the logic of this theory, then maximizing what is left over for shareholders is equivalent to maximizing the size of the whole pie. Thus shareholders always gain if the price of the stock goes up, but their potential losses are limited on the downside. In effect, creditors and other claimants are bearing some of the downside risk - they may be the ones who lose if the firm loses the gamble." (Blair 2003, 57)

Eine Legitimationsgrundlage für Residualgewinne auf Basis des Risikos aus ungesicherten spezifischen Investitionen lässt sich also mindestens genauso logisch plausibel für den Mitarbeiter wie für den Aktionär begründen.

11 Schauen wir uns einige Risikoelemente im Vergleich Aktionär versus Mitarbeiter genauer an: Betrachtet man z.B. die Diversifizierung als eine Massnahme zur Risikominderung, so fällt auf, dass Aktionäre ihr Risiko über geschicktes Portfoliomanagement diversifizieren können (Kaufman/Englander 2005, 16ff.). Mitarbeiter hingegen sind kaum in der Lage, Absicherungen in Form von Diversifizierungen durchzuführen (Boatright 2004, 12). Auch hinsichtlich des Investitionstyps und der Investitionsmenge zeigen sich Unterschiede. Der Aktionär ist in der Regel mit kurzfristig nicht benötigtem Geld teilinvestiert. Der Mitarbeiter hingegen ist mit Zeit und Arbeit voll investiert (nur bei Teilzeitarbeit trägt er ein geringeres Risiko). Während der Aktionär ferner mit Blick auf die Risikohöhe Gefahr läuft, einen materiellen (Teil-)Verlust zu erleiden (ein Totalverlust ist nur bei Konkurs des Unternehmens oder bei Derivaten möglich), steht bei dem Mitarbeiter der Arbeitsplatz auf dem Spiel (in der Regel Totalverlust, ausser bei Arbeitszeitverkürzung) (Boatright 2004, 12). Aktionäre haften im Gegensatz zum Mitarbeiter nur begrenzt für ihren finanziellen Einsatz. Betrachten wir die Sicherheit der vertraglichen Beziehung, so geht der Aktionär sein Investitionsrisiko bewusst ein und bekommt dafür auch höhere Renditen in Aussicht gestellt. Der Mitarbeiter sichert lediglich seine expliziten Verträge, die impliziten bleiben weiterhin risikoreich. Zwar können einzelne Aktionäre beim Kauf von Aktien keine Details verhandeln, wie dies etwa der Mitarbeiter in seinem Arbeitsvertrag kann (Boatright 1994, 398), eine Entscheidungsrevision wäre aber jederzeit möglich, da Unternehmen kontinuierlich Informationen an den Markt senden und der Aktionär im Gegensatz zum Mitarbeiter jederzeit die Exitoption wählen kann (Freeman/Evan 1990, 341). 
Diesen Grundgedanken bauen Blair, Kay und Stout in Anlehnung an die Theorie der Teamproduktion (Alchian/Demsetz 1972) und die Theorie unvollständiger Verträge (Zingales 1998; Tirole 1999 und 2001) aus und argumentieren ebenfalls für eine stärkere Berücksichtigung der Mitarbeiter (Kay 1995 und 1996; Blair/Stout 1999, 2000 und 2001). Ihrer Meinung nach ist die Position Williamsons und anderer Ökonomen, Aktionäre als alleinige residual claimants zu verstehen, keinesfalls die Regel: „(M)ost modern corporations do not fit the model underlying this analysis, and in practice, shareholders are rarely the only residual claimants." (Blair 1998, 196; vgl. dazu auch Blair 1995, 229ff.; Blair/Stout 1999, 275ff.) Spezifische Investitionen wie etwa Spezialkenntnisse oder Beziehungsnetzwerke stellen für den Mitarbeiter versunkene Kosten dar, die nur mit hohem Wertverlust alternativ reinvestiert werden können (z.B. in Form einer anderen Beschäftigung) (Blair 1995 sowie 2003, 58). Die Quasirente, die der Mitarbeiter als Differenz zur zweitbesten Alternative erhält, geht bei einem Scheitern der Kooperationsbeziehung verloren. Zwar wäre die Erfüllung der expliziten - nach Möglichkeit langfristigen - Verträge in der Regel juristisch einklagbar, ${ }^{12}$ dies gilt jedoch nicht für die impliziten Verträge, deren Umfang mit dem Grad der Spezifität ansteigt. Daraus ergibt sich in der Konsequenz, dass ungesicherte Risiken die Shareholder-Dominanz einschränken:

„More generally, from this incomplete contracting theoretical perspective, other contracting parties besides the stockholders are not fully protected by explicit contracting, thereby undermining the foundational premise of shareholders' supremacy. “ (Maboney et al. 2004, 20) ${ }^{13}$

Das Primat der Shareholder kann nur aufrechterhalten werden, wenn alternative Absicherungsmechanismen das ungesicherte Risiko auf Basis spezifischer Investitionen der Mitarbeiter auffangen.

\section{Absicherungsmechanismen gegen ungesicherte spezifische Investitionen von Mitarbeitern}

Im Folgenden werde ich drei solcher Absicherungsmechanismen vorstellen (Barca/Felli 1992; Blair 1998; Thomsen 2006, 43ff., und in Ansätzen Boatright 2004): (1) die Beteiligung am Eigentum (ownership), (2) die Mitwirkung an der Kontrolle (control) und (3) die Einhaltung von Versprechen, die sich aus der Einhaltung von impliziten Verträgen ergeben (promise). Während die ökonomische Standardliteratur sich insbesondere mit den ersten beiden Absicherungsmechanismen befasst (Berle/Means 1932), möchte ich insbesondere die dritte Alternative in den Blick nehmen und daraus meine Position begründen. Thomsen fasst die drei Alternativen wie folgt zusammen:

„The concerns of these critical stakeholders may be internalized in the corporate objective
function through other non-market governance mechanisms. First, the critical stakeholders
may acquire complete or partial ownership of the firm (internalization by ownership) as, for
example, in a management buy-out. Second, they may be directly, or indirectly, represented on
the company's board (internalization by board representation). Third, their preferences may be

12 Ausnahmen finden sich z.B. im Konkursfall, wenn auf Basis vertraglicher oder gesetzlicher Rahmenbedingungen Ansprüche nicht oder nicht vollständig erfüllt werden können.

13 Mahoney et al. greifen auf Zingales zurück: „If many members of the nexus [of contracts] are residual claimants, why are shareholders necessarily the ones affected the most by the firms' decisions? Even if they are, are they the party that benefits the most from the additional protection granted by the control rights?" (Zingales 2000, 1632, zit. nach Mahoney et al. 2004, 20) 
internalized by implicit contracts like reputation, culture or socialization [sic!]." (Thomsen 2004, 33)

Betrachten wir also zunächst in aller Kürze eine mögliche Beteiligung des Mitarbeiters am Eigentum (Hansmann 1996; Williamson 1996; Boatright 2004, 9ff.; Thomsen 2006, 43f.). Trotz einer weiten Verbreitung in der Praxis, gibt es auch kritische Positionen: So verfügen nicht alle Mitarbeiter über ausreichend Kapital, um sich am Unternehmen zu beteiligen, und wenn doch, sind Mitarbeiter in der Regel risikoavers. Ferner verfügt der Mitarbeiter über unzureichende Informationen, um das Unternehmen gut einschätzen zu können. Schliesslich führt eine Beteiligung des Mitarbeiters am Eigentum zu einer Verwässerung der Eigentumsrechte (Thomsen 2006, 43).

Auch bei der Kontrolle ergibt sich ein differenziertes Bild. Einige Autoren fordern eine employee governance, im Rahmen derer eine stärkere Beteiligung von Mitarbeitern an der Kontrolle möglich ist (Jacoby 2001; Boatright 2004). Neben den sich aus dem Eigentum ableitenden Wahlrechten (z.B. Wahl des Aufsichtsrats), können Stakeholderinteressen durch eine Beteiligung im Board vertreten werden. ${ }^{14}$ Die gesetzlich erzwungene Partizipation des Mitarbeiters im Kontrollgremium schützt die spezifische Investition des Mitarbeiters und verhindert, dass ein rationaler Mitarbeiter die hold-up-Gefahr antizipiert und entweder gar nicht oder auf einem ineffizienten Niveau in unternehmensspezifisches Humankapital investiert. Mitbestimmung ist in dieser Hinsicht insbesondere für Unternehmen mit einem hohen Anteil an spezifischem Humankapital profitabel. Dabei ist jedoch zu berücksichtigen, dass die Mitwirkung an der Kontrolle mit Kosten der Koordination und der Entscheidungsfindung und damit mit Effizienzverlusten verbunden sein kann (Hansmann 1996; Tirole 2001). Im Ergebnis führt dies dazu, dass - je nach Governancestruktur (one or two tier model of corporate governance) - lediglich Aktionäre und gegebenenfalls auch Mitarbeiter, weniger aber andere Stakeholder an der Kontrolle des Unternehmens beteiligt werden. ${ }^{15}$ Darüber hinaus ist eine hinreichende Kontrolle aufgrund von unvollständiger Information, Informationsasymmetrien und begrenzter Rationalität kaum möglich, so dass viele Autoren skeptisch sind: „(G)iven the breadth of our concept of stakeholder we believe that co-optation through participation is not always the correct strategic decision." (Freeman/Reed 1983, 92)

Weder ownership noch control sind also geeignete und effektive Massnahmen, Mitarbeiter an der Governance des Unternehmens zu beteiligen. Boatright bringt das wie folgt auf den Punkt:

„(T)he main traditional strategies for realizing employee governance, such as participatory management, employee stock ownership, and employee representation on boards of directors, are not likely to be effective." (Boatright 2004, 2f.)

14 Hier bieten sich drei Optionen an: (1) Stakeholder sind Mitglieder des Boards (representation of stakeholders on boards), (2) Stakeholder werden an den Ausschüssen beteiligt (representation on specific board committees) oder (3) es gibt einen eigenen Ausschuss, der nur aus Stakeholdern besteht (incorporation of stakeholderoriented committees within boards) (Thomsen 2006).

15 In den USA und in Grossbritannien gibt es für eine Partizipation von Nicht-Shareholdern an der Kontrolle des Unternehmens keine rechtliche Grundlage (Sadowski et al. 2003; McCall 2001). Insgesamt wird die Legitimation aufgrund von ungesicherten Quasirenten im angloamerikanischen Raum unter Juristen kontrovers diskutiert. In Deutschland hingegen wird die gesetzliche Mitbestimmung als staatlicher Eingriff in den Markt gerade dadurch gerechtfertigt, dass die transaktionsspezifische Quasirente nicht vollständig kontrahierbar ist. Dieser Umstand ist juristisch aber umstritten, da Unternehmen der Industriezweige mit einem geringen Anteil an spezifischem Humankapital versuchen könnten, gegen die gesetzliche Mitbestimmung gerichtlich vorzugehen. 
Während die ersten beiden Optionen dem Gedanken der Trennung von ownership and control folgen, nämlich den Mitarbeiter wie einen Aktionär zu behandeln, soll im Weiteren das Augenmerk auf die Absicherung von impliziten Verträgen durch das Einhalten von Versprechen als eine dritte mögliche Absicherungsvariante gelegt werden (Thomsen 2004, 33).

\section{Versprechen und treuhänderische Pflichten als dritte Absicherungsalternative}

Das Versprechen basiert auf der Übernahme treuhänderischer Pflichten und bildet eine dritte Schutzmassnahme für diejenigen Stakeholdergruppen, die aufgrund ihrer ex ante nicht geschützten unternehmensspezifischen Investition ein residuales Risiko tragen. „A third way for companies to internalise stakeholder concerns is to increase their creditability and trustworthiness through implicit contracts [...]." (Thomsen 2006, 46f.) Der implizite und unvollständige Vertrag ist eine besondere Form des Versprechens:

„The moralist of duty thus posits a general obligation to keep promises, of which the obligation of contract will be only a special case - that special case in which certain promises have attained legal as well as moral force. But since a contract is first of all a promise, the contract must be kept because a promise must be kept.“ (Fried 1981, 17)

Implizite und unvollständige Verträge bekommen vor diesem Hintergrund ebenso ein stärkeres Gewicht wie das gesprochene Wort in Form eines Versprechens (Atiyah 1981; Fried 1981; Kimel 2003). Letztlich geht es um individuelle Selbstbindung.

"The institution of promising is a way for me to bind myself to another so that the other may expect a future performance, and binding myself in this way is something that I may want to be able to do." (Fried 1981, 14)

Als Kooperationsversprechen bildet es einen neuen - jetzt sogar auch normativen - Koordinationsmechanismus, der gleichwertig neben Markt und Hierarchie ökonomische Transaktionen regelt (Coase 1937). Durch die Konvention wird deutlich, dass Versprechen stillschweigend gesellschaftliche Normen voraussetzen:

"The question now is not, how to make a promise, or how to use the facilities offered by the legal or social institution of promising, where one wishes quite deliberately to create an obligation; the question is whether one party, by his words and conduct, acted in such a way as to create an obligation." (Atiyah 1981, 170)

In diesem Zusammenhang sind zwei distinkte Konventionsebenen zu unterscheiden: Die erste Konventionsebene (linguistically conventional) besagt, dass es eine Übereinkunft darüber gibt, sich an sein gesprochenes Wort zu halten, und zwar unabhängig vom Inhalt des Versprechens. Man könnte also in Anlehnung an das pacta sunt servanda von einem promissiones sunt servandae sprechen: „(P)romises can sometimes be said to facilitate a form [sic!] of co-ordination between promisor and promisee. " (Kimel 2003, 9) Neben der eher formalen Koordinationsebene gibt es eine zweite, eine materiale Koordinationsebene, in der das konkrete Koordinationsproblem adressiert wird, wie zum Beispiel, auf welcher Strassenseite man fährt oder ob man sich bei einer Begrüssung die Hand reicht oder nicht. Die materiale Ebene ist jedoch für das Versprechen weniger relevant als für Konventionen im Allgemeinen.

Die ökonomische Standardposition negiert eine spezifisch moralische Verantwortung einzelner Boardmitglieder, die über fiduciary duties gegenüber Shareholdern hinausgeht (Clark 1985; Barca/Felli 1992; Hart 1993; Boatright 1994). In einer bekannten Auseinandersetzung im Harvard Law Review zwischen Dodd und seinem Schüler Berle wurde jedoch 
schon in den 30er Jahren des letzten Jahrhunderts die Frage nach den treuhänderischen Pflichten des Managements ausgiebig diskutiert. Während der Unternehmer seine Handlungen im Rahmen vertraglicher Abmachungen nur vor sich selbst zu verantworten hat, rekonstruiert Dodd den Manager als „fiduciary who must loyally serve his principal's interests“ (Dodd 1932, 1145). Berle leitet daraus ein treuhänderisches Verhältnis zwischen Prinzipal und Agent ab:

„Wherever one man or a group of men entrusted another man or group with the management of property, the second group became fiduciaries. As such they were obliged to act conscionably, which meant in fidelity to the interests of the persons whose wealth they had undertaken to handle." (Berle/Means 1932, 336) ${ }^{16}$

Berle fordert - aufgrund der Probleme zwischen Prinzipal und Agent - eine stärkere Kontrolle des Managements. Die Kontrolleure „function today more as princes and ministers than as promoters or merchants “ (Berle 1932, 1366f.). Zugleich registriert Berle einen stärkeren Kontrolleinfluss der Gesellschaft und der öffentlichen Meinung.

Diese Diskussion ist nun in zweierlei Hinsicht bemerkenswert. Zum einen relativiert sie die weit verbreitete Position, die auf Berle/Means zurückgehende Trennung von Eigentum und Kontrolle würde letztlich keinen Raum für eine gesellschaftliche oder unternehmensethische Verantwortung lassen. Zum anderen deutet sich an, dass die öffentliche Meinung Einfluss auf die Governancestrukturen im Unternehmen nimmt. Dodd schliesst in diesem Zusammenhang:

„A sense of social responsibility toward employees, consumers, and the general public may thus come to be regarded as the appropriate attitude to be adopted by those who are engaged in business, with the result that those who own their own businesses and are free to do what they like may increasingly adopt such an attitude. Business ethics may thus tend to become in some degree those of a profession rather than of a trade." (Dodd 1932,1160f.)

Dodd deutet in diesem Aufsatz bereits Absicherungsmöglichkeiten an, auf die sich unsere weitere Argumentation stützt:

„Experience may indicate that corporate managers are so closely identified with profit-seeking capital that we must look to other agencies to safeguard [sic!] the other interests involved [...].“ (Dodd 1932, 1161)

Einige Autoren setzen sich für eine spezifische Verantwortung des Boards ein, die sich in Form bestimmter ständiger Ausschüsse (standing committees, insbesondere standing ethics committees) oder in der Verantwortung des Boards in seiner Gesamtheit bzw. seiner einzelnen Mitglieder widerspiegelt (Freeman/Evan 1990; Evan/Freeman 1993; Driscoll/Hoffman 1994; Driscoll 1995; Luoma/Goodstein 1999; Felo 2001; Bonnafous-Boucher 2005; Schwartz et al. 2005). Empirische Studien von Felo (2001) etwa zeigen, inwieweit sich z.B. die Beteiligung von Boardmitgliedern an der Überwachung von Ethik-Programmen auf die Existenz und Verbreitung von Interessenkonflikten (z.B. CEO compensation und board composition) auswirkt. Im Ergebnis stellt der Autor heraus, dass Interessenkonflikte selte-

16 Ein solcher Standpunkt basiert auf der Annahme, Unternehmen seien eine legal fiction, bei der Verträge im eigentlichen Sinne zwischen den Anspruchsgruppen und den Eigentümern erfolgen (Dodd 1932, 1146). Jensen/ Meckling (1976) haben diesen Punkt aufgenommen, indem sie von einer multitude of contracts sprechen. Betrachtet man hingegen das Unternehmen als distinct legal person, so werden die Verträge mit dem Unternehmen selbst geschlossen und die Manager sind dem Unternehmen als Institution verantwortlich (vgl. z.B. Blair/ Stout 1999, 292). Die Verantwortung für die Aktionäre leitet sich dann nur indirekt ab. 
ner auftraten, wenn die Boardmitglieder aktiv an der Überwachung von Ethik-Programmen beteiligt waren (vgl. ähnlich auch Driscoll/Hoffman 1994).

Andere Autoren bezeichnen die treuhänderischen Pflichten als unvollkommene Pflichten (imperfect duties) im Sinne eines tugendethischen Verhaltens (Solomon 1992; Buchanan 1996). Und wieder Andere wie Schwartz et al. plädieren für freiwillige Selbstverpflichtungen von Aufsichtsräten. In ihrem Paper Tone at the Top diskutieren die Autoren einen eigenen Ethics Code for Directors (Schwartz et al. 2005): „Directors have overall responsibility for the ethics and compliance programs of the corporation." (Schwartz et al. 2005, 82) Ihre Argumentation basiert auf der Idee, dass rechtliche Vorschriften nicht ausreichen:

„(T)he legal system underlying corporate governance, although necessary, is inherently insufficient as a means of ensuring essential levels of ethical behavior on the part of corporate directors." (Schwartz et al. 2005, 81)

Nun könnte man behaupten, dass die Bindung an ein Versprechen wie z.B. in Form eines Berufsethos nur von professionalisierten Berufsgruppen wie Ärzten, Rechtsanwälten oder Wirtschaftsprüfern eingefordert werden kann. Es fällt jedoch auf, dass Ethik-Kodizes auch für andere Managergruppen aus Marketing, Finanzen oder Verkauf diskutiert und implementiert wurden. Der Ethics Code for Directors zum Beispiel umfasst Werte wie honesty, integrity, loyalty, responsibility, fairness und citizenship (Schwartz et al. 2005, 91ff.). Aus einer vertragstheoretischen Perspektive arbeitet Clark vier „legal attributes of the fiduciary relationship“ (Clark 1985, 71) heraus: (1) affirmative duties to disclosure, (2) open-ended duties to act, (3) closed-in rights to positional advantages und (4) moral rhetoric. Unter die closed-in rights to positional advantages fallen z.B. das Verbot von Eigenhandel (self-dealing), persönlicher Vorteilsnahme und Insiderhandel (Clark 1985, 74f.). Auch Blair und Stout befürworten etwa die Loyalität (duty of loyalty) oder die Fürsorge (care) als treuhänderische Pflicht:

„The duty of loyalty prohibits directors from various forms of self-dealing, including prohibiting them from taking a business opportunity or other property that belongs to the corporation.“ (Blair/Stout 2001, 429; vgl. auch Blair/Stout 1999, 298ff.)

Nach Blair/Stout sind Boardmitglieder keine agents der Aktionäre im eigentlichen Sinne, sondern übernehmen als trustees lediglich eine treuhänderische Funktion (autonomous fiduciaries) (Blair/Stout 1999, 290).

„The fact that the directors are not subject to any corporate participant's direct control does not mean, however, that directors are free to use this autonomy to promote their own interests. Rather than being agents, directors play a role that more closely resembles that of an autonomous trustee or fiduciary who is charged with serving another's interests. Thus numerous judicial opinions describe the director's role in fiduciary terms. " (Blair/Stout 2001, 424)

Das Unternehmen ist in diesem Sinne ein „nexus of firm-specific assets that have been invested by a variety of groups, including most obviously shareholders, bondholders, managers, and employees “ (Blair/Stout 2001, 425). Das Versprechen wird damit zu einem dritten Absicherungsmechanismus in Netzwerkorganisationen, dessen Ziel es ist, „to balance team members' competing interests in a fashion that keeps everyone happy enough that the productive coalition stays together" (Blair/Stout 1999, 281), und deren Struktur aus eher unvollständigen Verträgen besteht. Blair/Stout (2001) und Blair (2003) heben die unternehmensethischen Aspekte aus der teamproduktionstheoretischen Perspektive hervor und betonen, 
„that it is the special role of corporate directors, in this context, to be people whom the team members feel they can trust, the wise elders, persons of honor and integrity, as well as of wisdom and good judgment. Just as the board as a whole is part of an institutional arrangement to facilitate trust, board members must be seen as the keepers and upholders of the team's trust." (Blair 2003, 71)

Das Board als Kontrollorgan übernimmt damit letztlich die Gestaltung der Governanceund Absicherungsmechanismen (Blair/Stout 1999 und 2001; Osterloh/Frey 2005b).

„(I)t is the board which has to take over the task of governing the firm-specific investments and mediating between possible conflicting interests of financial and knowledge investors in firm-specific assets which cannot be contracted ex ante." (Osterloh/Frey 2005b, 10)

In diesem Sinne stellt das Board eine Überwachungsinstanz dar, die Absicherungsmechanismen von ungesicherten Risiken in einem Vertragsnetzwerk steuert.

\section{Diskussion der Ergebnisse und Ausblick}

Blair und Stout haben ihren bahnbrechenden Aufsatz im Virginia Law Review mit den Worten geschlossen, dass - ausgehend von erfolgreichen, aber auch irreführenden Ergebnissen aus der Prinzipal-Agent-Theorie - „(w)e are convinced that future debates about corporate governance will be more fruitful if they start from a better model that more accurately captures the fundamental contracting problem corporation law attempts to resolve. The mediating hierarchy model is a first step toward that better view." (Blair/Stout 1999, 328)

Netzwerkgovernance befasst sich mit der Führung, Steuerung und Kontrolle solcher Stakeholdernetzwerke. Spezifität ist die wesentliche Erklärungsvariable für normative Ansprüche, welche über die drei Absicherungsmechanismen Eigentum, Kontrolle oder Versprechen bedient werden können. Dadurch werden nicht nur Quasirenten gesichert und Stakeholderinteressen ausbalanciert, sondern häufig auch Unternehmenswerte geschaffen und Agenturkosten reduziert. Zwar sind auch die Absicherungsmechanismen nicht kostenfrei, dennoch liegt die Vermutung nahe, dass die Kosten der Absicherungsmechanismen durch Kostensenkungen an anderer Stelle kompensiert werden können wie zum Beispiel durch sinkende bonding und monitoring costs:

„Theoretically it is argued [...] that the agency costs [...] will probably decrease because of changing ownership of the residual risk [...].“ (Soppe 2008, 221)

Einige Autoren wie etwa Holmstrom/Roberts kritisieren die Fokussierung auf die Spezifität in der Ökonomik, wie sie von Williamson vertreten wird: „(T)he theory of the firm [...] has become too narrowly focused on the hold-up problem and the role of asset specificity." (Holmstrom/Roberts 1998, 91) Deshalb plädieren sie dafür, einen „much broader view of the firm and the determination of its boundaries " (Holmstrom/Roberts 1998, 75) einzunehmen. Zwar verweisen auch andere Quellen auf ein gleichermassen vorhandenes Risiko bei vielen unterschiedlichen Stakeholdergruppen (vgl. etwa Mahoney et al. 2004, Fussnote 4 und die darin enthaltene Literatur). Der Mitarbeiter wird aber immer einen Sonderstatus behalten, weil sein Vertragsverhältnis - im Gegensatz zu anderen Stakeholdergruppen - weniger aus expliziten und damit aus vollständigen Verträgen besteht, sondern zunehmend aus impliziten und damit unvollständigen Verträgen. Spezifisches Humankapital bildet sich in einer Wissensgesellschaft insbesondere in sozialen Netzwerken, welche durch Vertrauen bzw. durch die Einhaltung von Normen stabilisiert werden. Dennoch gilt grundsätzlich: 
„First, employees are important residual claimants especially when firm-specific human capital is involved. Second, creditors can be important residual claimants. Third, complex network relationships among industrial suppliers and customers produce interdependencies and lead to important residual gains and losses." (Mahoney et al. 2004, 14f.; vgl. auch Blair/Stout 1999, 276, Fussnote 61)

Bei der mittlerweile kaum mehr zu überblickenden Flut an Literatur zum Verhältnis des Unternehmens zu seinen Anspruchsgruppen ist eines jedoch offensichtlich: Die Theorie der Unternehmung ist aufgefordert, auch nach ihren normativen Grundlagen zu fragen und damit vor allem die in der Ökonomie scheinbar ex ante geklärte Legitimität von Corporate Governance neu zu erforschen:

„(F)or the first time in the history of the firm, or even of capitalism, rules of governance (considered as the designation of a sovereign power) are searching for a legitimizing instance originating outside the framework of those rules. “ (Bonnafous-Boucher 2005, 35)

Orientiert man sich an den Ansprüchen, die von Stakeholdern vorgetragen werden, deren unternehmensspezifische Investitionen nicht ex ante vertraglich geschützt wurden, so hat man eine „präzise und ökonomisch begründete Abgrenzung der anspruchsberechtigten Stakeholder zur Verfügung “ (Osterloh/Frey 2005a, 10) und Freemans Ansatz, dem oftmals Beliebigkeit vorgeworfen wird, bekommt ökonomische Schärfe. Der stake wird im vorliegenden Beitrag über die ungesicherten unternehmensspezifischen Investitionen rekonstruiert. Es geht nicht etwa darum, unterschiedliche Stakeholderinteressen auszubalancieren, sondern um konkret und ökonomisch gut begründete Stakeholderansprüche, denen man mit verschiedenen Governancemechanismen begegnet.

Zwar ist der hier vorgestellte Ansatz, ungesicherte spezifische Investitionen von Mitarbeitern stärker in den Blick zu nehmen und das Versprechen als Absicherungsmechanismus herzuleiten, an die Theorie der Unternehmung ökonomisch anschlussfähig, er weist jedoch mindestens zwei Schwachstellen auf: In der vorgestellten Theorie haben diejenigen Stakeholder einen legitimen Anspruch, die ungesichert spezifisch investiert sind. Ein gewisses Unbehagen bleibt hinsichtlich zweier Punkte: (1) Wie werden diejenigen Anspruchsgruppen behandelt, die unspezifisch investiert sind, und (2) wie diejenigen, die gar nicht investiert sind? Normative Stakeholdertheorien konnten bisher keine zufriedenstellenden Antworten darauf geben und wurden in der Literatur heftig kritisiert (Hendry 2001, 162; Phillips 2003). ${ }^{17}$ Philosophen, denen man in normativen Fragen einen fundierten Sachverstand zutraut, haben in den Managementwissenschaften mit normativen Stakeholderkonzepten ebenfalls bislang keinen akzeptablen Durchbruch unter Governancetheoretikern geschafft:

„Despite all the attention given to normative stakeholder theory over the past decade, no-one within the business ethics community has made the case for a realistic version [sic!] of the theory such as might provide a viable practical alternative to the shareholder perspective. Meanwhile, despite all the talk of stakeholders, they have become increasingly marginal to the corporate governance debate, which has focused more and more narrowly on the two constituencies of shareholders and management and on the narrowly defined economic agency relationship that is supposed to bind them." (Hendry 2001, 173)

17 Das Stakeholdermanagement wurde in verschiedensten Varianten normativ erweitert (vgl. insbesondere Freeman/Reed 1983; Freeman/Evan 1990; Evan/Freeman 1993; Bowie/Freeman 1992; McCall 2001; Donaldson/ Preston 1995 sowie Phillips 1997 und 2003). 
Betrachten wir also zunächst den ersten Problemkreis: Eine unspezifisch investierte Anspruchsgruppe erzielt keine Quasirente, da die Differenz des Ertrages einer Investition in ihrer bestmöglichen Verwendung zum Ertrag in ihrer zweitbesten Verwendung gleich null ist. Folglich haben unspezifisch investierte Stakeholder weder einen Anspruch auf eine Beteiligung am Eigentum noch auf eine Beteiligung an der Kontrolle. Der zweite Problemkreis ist differenzierter zu betrachten. Stakeholder, die gar nicht in das Unternehmen investiert sind, also weder spezifisch noch unspezifisch, werden im Folgenden als non-Stakeholder bezeichnet (vgl. ähnlich Phillips 2003, 26). Sie haben keine versunkenen Kosten, erzielen keine Quasirente und aufgrund des non-Investments fallen in der Regel keine Transaktionskosten an. Nicht nur aus Sicht der Property-Rights-Theorie, die sich mit der Eigentums- und der Kontrollfrage befasst, sondern auch aus normativen Überlegungen scheint es zunächst plausibel, dass es keinen legitimen Anspruch für non-Stakeholder weder auf eine Beteiligung am Eigentum noch auf eine Beteiligung an der Kontrolle gibt. Solche Ansprüche würden das einzelne Unternehmen überfordern, wären nicht hinreichend legitimiert und ökonomisch nicht anschlussfähig.

Wie aber sieht es mit dem dritten Governance- bzw. Absicherungsmechanismus aus, dem Versprechen? Intuitiv würde man wohl keine Gesellschaft anstreben wollen, die in letzter Konsequenz bestimmte gesellschaftliche Gruppen vernachlässigt, zum Bespiel solche, die von einer spezifischen oder unspezifischen Investition prinzipiell ausgeschlossen sind (Kinder, Alte etc.) oder aber deren Chancen gering sind, zu investieren (Kranke, Bildungsschwache etc.). Ausserdem gibt es Anspruchsgruppen, die unverschuldet in Notlagen geraten sind und sich aus eigener Kraft nicht helfen können (Opfer von Überschwemmungen, Erdbeben, Anschlägen, Hunger, schweren und seltenen Krankheiten in der Dritten Welt etc.). Diese Anspruchsgruppen sind dann sozusagen unfreiwillig nicht investiert. Unternehmerische Verantwortung liesse sich in diesem Fall wohl nur aus sozialvertraglichen oder kantianischen Argumenten ableiten (dazu z.B. Donaldson/Dunfee 1994 und 1999). Die Forschung sollte sich diesen Themen intensiver widmen.

\section{Literaturhinweise}

Alchian, A. A./Demsetz, H. (1972): Production, Information Costs, and Economic Organization, in:

American Economic Review, Jg. 62, Nr. 5, S. 777-795.

Atiyah, P. S. (1981): Promises, Morals, and Law, Oxford.

Barca, F./Felli, L. (1992): Fiduciary Duties, Ownership, and Control, London.

Barnard, Ch. I. (1938): The Functions of the Executive, Cambridge.

Becker, G. S. (1964): Human Capital, Chicago.

Berle, A. A. (1932): For Whom Corporate Managers Are Trustees: A Note, in: Harvard Law Review, Jg. 45, Nr. 7, S. 1365-1372.

Berle, A. A./Means, G. C. (1932): The Modern Corporation and Private Property, New York.

Blair, M. M. (1995): Ownership and Control: Rethinking Corporate Governance for the Twenty-First Century, Washington.

Blair, M. M. (1998): For Whom Should Corporations Be Run? An Economic Rationale for Stakeholder Management, in: Long Range Planning, Jg. 31, Nr. 2, S. 195-200.

Blair, M. M. (2003): Shareholder Value, Corporate Governance, and Corporate Performance, in: Cornelius, P. K./Kogut, B. (Hrsg.): Corporate Governance and Capital Flows in a Global World, New York, Oxford, S. 53-82.

Blair, M. M./Stout, L. A. (1999): A Team Production Theory of Corporate Law, in: Virginia Law Review, Jg. 85, Nr. 2, S. 247-328. 
Blair, M. M./Stout, L. A. (2000): Trust, Trustworthiness and the Behavioral Foundations of Corporate Law, in: University of Pennsylvania Law Review, Jg. 149, Nr. 6, S. 1735-1810.

Blair, M. M./Stout, L. A. (2001): Director Accountability and the Mediating Role of the Corporate Board, in: Washington University Law Quarterly, Jg. 79, Nr. 2, S. 403-449.

Boatright, J. R. (1994): Fiduciary Duties and the Shareholder-Management Relation: Or, What's So Special About Shareholders?, in: Business Ethics Quarterly, Jg. 4, Nr. 4, S. 393-407.

Boatright, J. R. (2004): Employee Governance and the Ownership of the Firm, in: Business Ethics Quarterly, Jg. 14, Nr. 1, S. 1-21.

Bonnafous-Boucher, M. (2005): Some Philosophical Issues in Corporate Governance: The Role of

Property in Stakeholder Theory, in: Corporate Governance, Jg. 5, Nr. 2, S. 34-47.

Bowie, N. E./Freeman, R. E. (1992) (Hrsg.): Ethics and Agency Theory: An Introduction, New York.

Buchanan, A. (1996): Perfecting Imperfect Duties: Collective Action to Create Moral Obligations, in: Business Ethics Quarterly, Jg. 6, Nr. 1, S. 28-41.

Buchanan, J. M. (1964): Is Economics the Science of Choice?, in: Streissler, E. (Hrsg.): Roads to Freedom: Essays in Honor of F. A. Hayek, London, S. 47-64.

Buchanan, J. M. (1975): A Contractarian Paradigm for Applying Economic Theory, in: American Economic Review, Jg. 65, Nr. 2, S. 225-230.

Calton, J. M./Lad, L. J. (1995): Social Contracting as a Trust-Building Process of Network Governance, in: Business Ethics Quarterly, Jg. 5, Nr. 2, S. 271-295.

Che, Y.-K./Hausch, D. B. (1999): Cooperative Investments and the Value of Contracting, in: American Economic Review, Jg. 89, Nr. 1, S. 125-147.

Clark, R. C. (1985): Agency Costs versus Fiduciary Duties, in: Pratt, J./Zeckhauser, R. (Hrsg.): Principals and Agents: The Structure of Business, Boston, S. 55-79.

Clarkson, M. B. E. (1995): A Risk Based Model of Stakeholder Theory. Proceedings of the Second Toronto Conference on Stakeholder Theory, Centre for Corporate Social Performance \& Ethics, Toronto.

Coase, R. H. (1937): The Nature of the Firm, in: Economica, Jg. 4, Nr. 16, S. 386-405.

Dodd, E. M. Jr. (1932): For Whom Are Corporate Managers Trustees?, in: Harvard Law Review, Jg. 45, Nr. 7, S. 1145-1163.

Donaldson, T./Dunfee, T. W. (1994): Toward a Unified Conception of Business Ethics: Integrative Social Contracts Theory, in: Academy of Management Review, Jg. 19, Nr. 2, S. 252-284.

Donaldson, T./Dunfee, T. W. (1999): Ties That Bind: A Social Contracts Approach to Business Ethics, Cambridge.

Donaldson, T./Preston, L. E. (1995): The Stakeholder Theory of the Corporation: Concepts, Evidence, and Implications, in: Academy of Management Review, Jg. 20, Nr. 1, S. 65-91.

Driscoll, D. M. (1995): The Ethical Responsibility of Directors and Trustees, in: Montana Business Quarterly, Jg. 33, Nr. 3, S. 10.

Driscoll, D. M./Hoffman, W. M. (1994): Doing the Right Thing: Business Ethics and Boards of Directors, in: Director's Monthly, Jg. 18, Nr. 11 [Special Issue on Ethics], S. 1-7.

Evan, W. M./Freeman, R. E. (1993): A Stakeholder Theory of the Modern Corporation: Kantian Capitalism, in: Beauchamp, T. L./Bowie, N. E. (Hrsg.): Ethical Theory and Business, Englewood Cliffs, S. 97-106.

Felo, A. J. (2001): Ethics Programs, Board Involvement, and Potential Conflicts of Interest in Corporate Governance, in: Journal of Business Ethics, Jg. 32, Nr. 3, S. 205-218.

Fontrodona, J./Sison, A. J. G. (2006): The Nature of the Firm, Agency Theory and Shareholder Theory: A Critique from Philosophical Anthropology, in: Journal of Business Ethics, Jg. 66, Nr. 1, S. 33-42. 
Freeman, R. E. (1984): Strategic Management: A Stakeholder Approach, Boston.

Freeman, R. E./Evan, W. M. (1990): Corporate Governance: A Stakeholder Interpretation, in: The Journal of Behavioral Economics, Jg. 19, Nr. 4, S. 337-359.

Freeman, R. E./Reed, D. L. (1983): Stockholders and Stakeholders: A New Perspective on Corporate Governance, in: California Management Review, Jg. 25, Nr. 3, S. 88-106.

Fried, C. (1981): Contract as Promise. A Theory of Contractual Obligation, Cambridge.

Friedman, M. (1970): The Social Responsibility of Business Is to Increase Its Profits, in: New York Times Magazine, September 13, S. 32-33, 122, 124, 126.

Furubotn, E. G./Richter, R. (2005): Institutions \& Economic Theory. The Contribution of the New Institutional Economics, 2. Aufl., Ann Arbor.

Gathmann, C./Schönberg, U. (2010): How General Is Human Capital? A Task-Based Approach, in: Journal of Labor Economics, Jg. 28, Nr. 1, S. 1-49.

Ghoshal, S. (2005): Bad Management Theories Are Destroying Good Management Practices, in: Academy of Management Learning \& Education, Jg. 4, Nr. 1, S. 75-91.

Ghoshal, S./Moran, P. (1996): Bad for Practice: A Critique of the Transaction Cost Theory, in: Academy of Management Review, Jg. 21, Nr. 1, S. 13-47.

Hansmann, H. (1996): The Ownership of Enterprise, Cambridge.

Hart, O. (1993): An Economist's View of Fiduciary Duty, in: University of Toronto Law Journal, Jg. 43, Nr. 3, S. 299-313.

Heath, J./Norman, W. (2004): Stakeholder Theory, Corporate Governance and Public Management: What Can the History of State-Run Enterprises Teach Us in the Post-Enron Era?, in: Journal of Business Ethics, Jg. 53, Nr. 3, S. 247-265.

Hendry, J. (2001): Missing the Target: Normative Stakeholder Theory and the Corporate Governance Debate, in: Business Ethics Quarterly, Jg. 11, Nr. 1, S. 159-176.

Hill, C. W. L./Jones, T. M. (1992): Stakeholder-Agency Theory, in: Journal of Management Studies, Jg. 29, Nr. 2, S. 131-154.

Holmstrom, B./Roberts, J. (1998): The Boundaries of the Firm Revisited, in: Journal of Economic Perspectives, Jg. 12, Nr. 3, S. 73-94.

Jacoby, S. M. (2001): Employee Representation and Corporate Governance: A Missing Link?, in: University of Pennsylvania Journal of Labor and Employment Law, Jg. 3, Nr. 3, S. 449-490.

Jensen, M. C. (2001): Value Maximization, Stakeholder Theory, and the Corporate Objective Function, in: Journal of Applied Corporate Finance, Jg. 14, Nr. 3, S. 8-21.

Jensen, M. C./Meckling, W. H. (1976): Theory of the Firm: Managerial Behavior, Agency Costs and Ownership Structure, in: Journal of Financial Economics, Jg. 3, Nr. 1, S. 305-360.

Jones, C./Hesterly, W. S./Borgatti, St. P. (1997): A General Theory of Network Governance: Exchange Conditions and Social Mechanism, in: Academy of Management Review, Jg. 22, Nr. 4, S. 911945.

Jones, T. M. (1995): Instrumental Stakeholder Theory: A Synthesis of Ethics and Economics, in: Academy of Management Review, Jg. 20, Nr. 2, S. 404-437.

Kaufman, A./Englander, E. (2005): A Team Production Model of Corporate Governance, in: Academy of Management Executive, Jg. 19, Nr. 3, S. 9-22.

Kay, J. (1995): Why Firms Succeed, New York.

Kay, J. (1996): The Business of Economics, Oxford et al.

Kimel, D. (2003): From Promise to Contract. Towards a Liberal Theory of Contract, Oxford et al. Klein, B./Crawford, R. G./Alchian, A. A. (1978): Vertical Integration, Appropriable Rents, and the Competitive Contracting Process, in: Journal of Law and Economics, Jg. 21, Nr. 2, S. 297-326. 
Luoma, P./Goodstein, J. (1999): Stakeholders and Corporate Boards: Institutional Influence on Board Composition and Structure, in: Academy of Management Journal, Jg. 42, Nr. 5, S. 553-563.

Mahoney, J./Asher, C. C./Mahoney, J. (2004): Towards a Property Rights Foundation for a Stakeholder Theory of the Firm, Working Paper No. 04-0116, University of Illinois.

McCall, J. J. (2001): Employee Voice in Corporate Governance: A Defense of Strong Participation Rights, in: Business Ethics Quarterly, Jg. 11, Nr. 1, S. 195-213.

Osterloh, M./Frey, B. S. (2005a): Corporate Governance: Eine Prinzipal-Agenten-Beziehung, TeamProduktion oder ein Soziales Dilemma?, Zürich.

Osterloh, M./Frey, B. S. (2005b): Shareholders Should Welcome Employees as Directors, Arbeitspapier der Universität Zürich, http://ssrn.com/abstract=655202, Abfragedatum 08.09.2010.

Phillips, R. A. (1997): Stakeholder Theory and a Principle of Fairness, in: Business Ethics Quarterly, Jg. 7, Nr. 1, S. 51-66.

Phillips, R. A. (2003): Stakeholder Legitimacy, in: Business Ethics Quarterly, Jg. 13, Nr. 1, S. 25-41.

Quinn, D. P./Jones, T. M. (1995): An Agent Morality View of Business Politics, in: Academy of Management Review, Jg. 20, Nr. 1, S. 22-42.

Rajan, R. G./Zingales, L. (1998): Power in a Theory of the Firm, in: The Quarterly Journal of Economics, Jg. 113, Nr. 2, S. 387-432.

Roberts, J./van den Steen, E. (2000): Shareholder Interests, Human Capital and Corporate Governance, GSB Research Paper No. 1631, Stanford.

Ross, S. (1973): The Economic Theory of Agency: The Principal's Problem, in: American Economic Review, Jg. 63, Nr. 2, S. 134-139.

Sadowski, D./Junkes, J./Lindenthal, S. (2003): Labour Co-Determination and Corporate Governance in Germany: The Economic Impact of Marginal and Symbolic Rights, in: Schwalbach, J. (Hrsg.): Corporate Governance. Essays in Honor of Horst Albach, 2. Aufl., Berlin, S. 144-160.

Schwartz, M. S./Dunfee, T. W./Kline, M. J. (2005): Tone at the Top: An Ethics Code for Directors?, in: Journal of Business Ethics, Jg. 58, Nr. 1-3, S. 79-100.

Shankman, N. A. (1999): Reframing the Debate Between Agency and Stakeholder Theories of the Firm, in: Journal of Business Ethics, Jg. 19, Nr. 4, S. 319-334.

Shleifer, A./Vishny, R. W. (1997): A Survey of Corporate Governance, in: The Journal of Finance, Jg. 52, Nr. 2, S. 737-783.

Solomon, R. C. (1992): Ethics and Excellence. Cooperation and Integrity in Business, Oxford.

Soppe, A. (2008): Sustainable Finance and the Stakeholder Equity Model, in: Cowton, Chr./Haase, M.

(Hrsg.): Trends in Business and Economic Ethics, Berlin, Heidelberg, S. 199-228.

Suchman, M. C. (1995): Managing Legitimacy: Strategic and Institutional Approaches, in: Academy of Management Review, Jg. 20, Nr. 3, S. 571-610.

Thomsen, S. (2004): Corporate Values and Corporate Governance, in: Corporate Governance, Jg. 4, Nr. 4, S. 29-46.

Thomsen, S. (2006): Corporate Governance and Corporate Social Responsibility, in: Kakabadse, A./ Morsing, M. (Hrsg.): Corporate Social Responsibility: Reconciling Aspiration with Application, Basingstoke et al., S. 40-54.

Tirole, J. (1999): Incomplete Contracts: Where Do We Stand?, in: Econometrica, Jg. 67, Nr. 4, S. 741781.

Tirole, J. (2001): Corporate Governance, in: Econometrica, Jg. 69, Nr. 1, S. 1-34.

Wieland, J. (2007): Idealistische, ideale und reale Diskurse. Governanceformen des Diskurses, in: Wieland, J. (Hrsg.): Governanceethik und Diskursethik - ein zwangloser Diskurs, Marburg, S. $13-57$. 
Wieland, J. (2008): Governanceökonomik: Die Firma als Nexus von Stakeholdern. Eine Diskussionsanregung, in: Wieland, J. (Hrsg.): Die Stakeholder-Gesellschaft und ihre Governance. Management, Netzwerke, Diskurse, Marburg, S. 15-38.

Williamson, O. E. (1984a): Corporate Governance, in: Yale Law Journal, Jg. 93, Nr. 7, S. 1197-1230.

Williamson, O. E. (1984b): The Economics of Governance - Framework and Implications, in: Journal of Institutional and Theoretical Economics, Jg. 140, S. 195-223.

Williamson, O. E. (1985): The Economic Institutions of Capitalism: Firms, Markets, Relational Contracting, New York.

Williamson, O. E. (1990/1996): Chester Barnard and the Incipient Science of Organization, in: Williamson, O. E. (Hrsg.): The Mechanisms of Governance, Oxford et al., S. 29-53.

Williamson, O. E. (1996) (Hrsg.): The Mechanisms of Governance, Oxford et al.

Williamson, O. E. (1998): Transaction Cost Economics: How It Works; Where It Is Headed, in: De Economist, Jg. 146, Nr. 1, S. 23-58.

Williamson, O. E. (2002): The Theory of the Firm as Governance Structure: From Choice to Contract, in: Journal of Economic Perspectives, Jg. 16, Nr. 3, S. 171-195.

Williamson, O. E. (2005): The Economics of Governance, in: American Economic Review, Jg. 95, Nr. 2, S. 1-18.

Zingales, L. (1998): Corporate Governance, in: Newman, P. (Hrsg.): The New Palgrave Dictionary of Economics and the Law, New York, S. 497-503.

Zingales, L. (2000): In Search of New Foundations, in: Journal of Finance, Jg. 55, Nr. 4, S. 16231653.

Alexander Brink, Dr. Dr., ist Professor für Wirtschafts- und Unternehmensethik an der Universität Bayreuth, permanenter Gastprofessor für Corporate Governance \& Philosophy an der Privaten Universität Witten/Herdecke und Partner der von ihm mitgegründeten concern GmbH, einer CSR Beratungs- und Beteiligungsgesellschaft mit Sitz in Köln.

Anschrift: Universität Bayreuth, Institut für Philosophie, Universitätsstr. 30, D-95440 Bayreuth, Tel.: +49 (0)921/55-4122, Fax: +49 (0)921/55-4160, E-Mail: alexander.brink@unibayreuth.de 